\title{
Transport Properties Calculation for a Quasi-Bidimensional System using T-Matrix Approximation
}

\author{
C. P. Moca* and E. Macocian \\ Department of Physics, University of Oradea \\ 3700, Oradea, Romania
}

November 19, 2018

\begin{abstract}
We performed a self-consistent calculation using T-Matrix approximation for a quasi-bidimensional system. We calculated the one particle spectrum function $A(\mathbf{k}, \omega)$ in the presence of strong d-wave attractive interaction. The $c$-axis charge dynamics was studied by considering incoherent interlayer hopping and $a b$-plane charge dynamics was studied in the coherent limit. It is shown that the $c$-axis charge dynamics is mainly governed by the scattering from the in plane fluctuations. We also present results for $c$-axis and $a b$-plane resistivity and for thermopower coefficient.

Keywords: pseudogap, charge dynamics, resistivity, thermopower
\end{abstract}

\section{Introduction}

In recent years one of the significant advances in the research of high- $\mathrm{T}_{c}$ superconductors is the detailed experiments on the pseudogap behavior in underdoped high- $\mathrm{T}_{c}$ superconductors. The pseudogap phenomena have been recognized as one of the most important issues. There are enormous studies from both experimental and theoretical point of view. Influence of the pseudogap formation shows up in different experimental probes, such as NMR relaxation time, Knight shift, neutron scattering, tunneling, photoemission, specific heat, optical conductivity and d.c. resistivity[1]].

The pseudogap phenomena means the suppression of the low frequency spectral weight without any long range order. For cuprate superconductors the low frequency spectral weight begins to be strongly suppressed below some characteristic temperature $\mathrm{T}^{*}$ much higher than $\mathrm{T}_{c}$. Typically $\mathrm{T}^{*}$ is several times larger that $\mathrm{T}_{c}$ in underdoped cuprates and their doping dependence is qualitatively different. While $\mathrm{T}_{c}$ deceases with underdoping, $\mathrm{T}^{*}$ increases in contrast. This suggest that $\mathrm{T}^{*}$ does not follow the real $\mathrm{T}_{c}$ line but instead some kind of mean-field critical temperature $\mathrm{T}_{M F}$.

In reality the origin of pairing interaction should be considered to be the antiferromagnetic spin fluctuations. There are studies dealing with pairing correlations obtained by the spin fluctuations on the basis of the fluctuation-exchange (FLEX) approximation [2]. To explain the pseudogap phenomena many theories have been proposed, such as the argument based on BCS-BE cross-over [3], spin-charge separation induced by stripe order [4], RVB theory [5], strong superconducting phase fluctuations [6, 7], magnetic scenario near the antiferromagnetic instability [8, 9, 10] and formation of charge density waves (CDW) or spin density waves (SDW) [11]. A feature of the last scenario is that the pseudogap can persist in the superconducting state [12]. The effect of the magnetic field on the pseudogap in high- $\mathrm{T}_{c}$ cuprates has been theoretically investigated by Yanase et.al. 13] considering the Landau quantization for the superconducting fluctuations

\footnotetext{
${ }^{*}$ Corresponding author. Fax +40-59-432789;E-mail:mocap@ff.uoradea.ro
} 
as the main effect of the magnetic field. The pseudogap phenomena was studied in the context of non-Fermi Anderson model in [14] considering the electron-fluctuation interaction. Recent dimensional crossover study by Preosti et.al. [15] shows that the pseudogap effect is basically absent in three dimensions.

In this paper we describe the pairing scenario based on the strong coupling superconductivity. Generally the strong coupling superconductivity indicates the existence of the incoherent Cooper pairs as the Nozieres and Schmitt-Rink formalism [16]. We think of the pseudogap as the phenomena brought about by the strong superconducting fluctuations. Maly et.al. [17] have introduced the idea of "resonances" which means the scattering mechanism by the presence of metastable preformed pairs.

Through several years of extensive experimental work general consensus regarding the superconducting gap symmetry seems to be reach, that the superconducting gap has mainly $d$-wave character with possibility of a small mixture of other angular momentum state [18, 19, 20]. A remarkable point of the pseudogap is that its structure in momentum space is the same as the superconducting $d_{x^{2}-y^{2}}$ symmetry with continuous evolution through $\mathrm{T}_{c}$. This implies that the pseudogap phenomena has close connection to the superconducting fluctuations [21]. On the other hand using Intrinsic Tunneling Spectroscopy, Krasnov et.al. [22] found that the pseudogap is coexisting with the superconducting gap which imply that the two phenomena are different in nature. The precursor superconductivity scenario can not explain these experimental data and a possible explication might be the formation of CDW or SDW [11]. Another striking characteristic of cuprate superconductors is the anisotropy of their structure. The quantity which most evidently displays the anisotropy of a particular material is the optical conductivity $\sigma(\omega, T)$ and the corresponding d.c. resistivity $\rho(T)$ 23].

The opening of the pseudogap has drastic effect on the physical properties of the high- $\mathrm{T}_{c}$ cuprates [1]. It is found that associated with the pseudogap the in-plane resistivity deviates from the $T$-linear behavior [24] and the $\mathrm{T}$ coefficient of the $c$-axis resistivity changes sign, showing semiconducting behavior at low temperature 25.

This paper is constructed as follow. In section 2 we give the model Hamiltonian and explain the theoretical framework. In section 3 we present the results obtained for the spectral function $A(\mathbf{k}, \omega)$ and the results for the transport properties studied in this paper. In section 4 we summarize the obtained results and give conclusions.

\section{Theoretical Framework}

\subsection{Model Hamiltonian}

In this section we describe the theoretical framework of this paper. We consider a microscopic model which incorporates both strong electron-fluctuation interaction in the $\mathrm{CuO}$ planes and a weak interlayer coupling. Within each layer we consider the following two-dimensional model Hamiltonian which has $d_{x^{2}-y^{2}}$-wave superconducting ground state:

$$
H_{l}=\sum_{\mathbf{k}, \sigma} \varepsilon_{\mathbf{k}} c_{l \mathbf{k} \sigma}^{+} c_{l \mathbf{k} \sigma}+\sum_{\mathbf{q}, \mathbf{k}, \mathbf{k}^{\prime}} V_{\mathbf{k} \mathbf{k}^{\prime}} c_{l \mathbf{k} \uparrow}^{+} c_{l \mathbf{k}+\mathbf{q} \uparrow} c_{l \mathbf{k}^{\prime} \downarrow}^{+} c_{l \mathbf{k}^{\prime}-\mathbf{q} \downarrow}
$$

where $c_{l \mathbf{k} \sigma}$ is the annihilation operator for an electron with momentum $\mathbf{k}$ and $\operatorname{spin} \sigma$ in the layer $l$. The electron dispersion relation is expressed as:

$$
\varepsilon_{\mathbf{k}}=-2 t\left(\cos k_{x}+\cos k_{y}\right)-4 t^{\prime} \cos k_{x} \cos k_{y}-\mu
$$

where $t$ and $t^{\prime}$ are the nearest and next-nearest neighbors hopping amplitudes and $\mu$ is the chemical potential. From now on we will consider $t$ equal to unity and $t^{\prime}=-0.5 t$. The pair interaction is written as:

$$
V_{\mathbf{k k}^{\prime}}=V f_{\mathbf{k}} f_{\mathbf{k}^{\prime}}
$$


where

$$
f_{\mathbf{k}}=\cos k_{x}-\cos k_{y}
$$

is the $d_{x^{2}-y^{2}}$-wave form factor. In the previous relation $\mathrm{V}$ is negative. The hopping between layers is included in:

$$
H=\sum_{l} H_{l}-t_{c} \sum_{l}\left(c_{l i \sigma}^{+} c_{l+1 i \sigma}+c_{l+1 i \sigma}^{+} c_{l i \sigma}\right)
$$

where $t_{c}$ is the hopping amplitude between layers and $c_{l i \sigma}$ is the annihilation operator for an electron within the planar site $i$, with spin $\sigma$ in the layer $l$. The important character of high- $\mathrm{T}_{c}$ cuprates is the momentum dependence of the interlayer hopping matrix element $t_{c}(\mathbf{k})$. The band calculations [26, 27] of these materials have shown that the c-axis tunneling matrix element is larger at $(\pi, 0)$ and symmetry related points. The functional form is:

$$
t_{c}(\mathbf{k})=\left(\cos k_{x}-\cos k_{y}\right)^{2}
$$

On the other side the coupling between layers has little effect on the pseudogap 28]. The out of plane resistance is determined mainly by the properties of individuals $C u O$ layers. The large magnitude of the resistivity anisotropy reflects that the $c$-axis mean free path is shorter than the interlayer distance, and the carriers are tightly confined to the $\mathrm{CuO}$ planes and also is the evidence of the incoherent charge dynamics in the $c$-axis direction.

The interlayer part of the Hamiltonian (5) will be considered for the calculation of $c$-axis charge dynamics and $\rho_{c}(T)$.

\subsection{Self-Consistent T-Matrix Approximation}

In this paper we focus on the calculation of the Green function directly on the real-frequency axis [29] in order to avoid the difficulties of controlling the accuracy of the calculations used in the numerical analytical continuations from the imaginary to real axis by Pade algorithm [30].

We carry out a self-consistent calculation for the spectral function:

$$
A(\mathbf{k}, \omega)=-\frac{1}{\pi} \operatorname{Im} G(\mathbf{k}, \omega)
$$

where the retarded Green function $G(\mathbf{k}, \omega)$ is given by:

$$
G(\mathbf{k}, \omega)=\frac{1}{\omega-\varepsilon_{\mathbf{k}}-\Sigma(\mathbf{k}, \omega)}
$$

where $\Sigma(\mathbf{k}, \omega)$ is the retarded self-energy. The imaginary part of the self-energy can be expressed as:

$$
\operatorname{Im} \Sigma(\mathbf{k}, \omega)=f_{\mathbf{k}}^{2} \sum_{\mathbf{k}^{\prime}} \int d \omega^{\prime}\left[f\left(\omega^{\prime}\right)+n\left(\omega+\omega^{\prime}\right)\right] A\left(\mathbf{k}^{\prime}, \omega^{\prime}\right) \operatorname{Im} T\left(\mathbf{k}+\mathbf{k}^{\prime}, \omega+\omega^{\prime}\right)
$$

where $f(\omega)$ and $n(\omega)$ represent Fermi-Dirac and Bose-Einstein distributions. The real part of the retarded self-energy can be calculated directly or using Kramers-Kronig relation:

$$
\operatorname{Re} \Sigma(\mathbf{k}, \omega)=p . v \cdot \int \frac{d \omega^{\prime}}{\pi} \frac{\operatorname{Im} \Sigma\left(\mathbf{k}, \omega^{\prime}\right)}{\omega-\omega^{\prime}}
$$

where p.v. represents the principal value of the integral. The Hartree term is neglected in the self-energy. The T-Matrix is given by the following relation:

$$
T(\mathbf{k}, \omega)=\frac{V^{2} \Pi(\mathbf{k}, \omega)}{1+V \Pi(\mathbf{k}, \omega)}
$$


where $\Pi(\mathbf{k}, \omega)$ can be evaluated directly from the spectral function as:

$$
\operatorname{Im} \Pi(\mathbf{q}, \omega)=\pi \sum_{\mathbf{k}} \int d \omega^{\prime} f_{\mathbf{k}}^{2} \tanh \frac{\omega}{2 T} A\left(\mathbf{k}, \omega^{\prime}\right) A\left(\mathbf{q}-\mathbf{k}, \omega-\omega^{\prime}\right)
$$

The chemical potential is determined by fixing the density $n$ through the relation:

$$
\frac{n}{2}=\sum_{\mathbf{k}} \int d \omega A(\mathbf{k}, \omega) f(\omega)
$$

We can calculate the hole doping $\delta$ using the relation $\delta=1-n$. Beside relation (13) we must take care of the sum-rule satisfied by the spectral function:

$$
1=\sum_{\mathbf{k}} \int d \omega A(\mathbf{k}, \omega)
$$

which must be evaluated at each step in the self-consistent calculation. In the present calculation we divided first Brilouinne zone into $32 \times 32$ or $64 \times 64$ lattice and the frequency integration was replaced by a discrete sum of 1024 or 512 points. We did not found differences in the behavior of spectral function using these two discretizations.

Performing the self-consistent calculation given by Eqs. (7)-(14) we obtain the spectral function $A(\mathbf{k}, \omega)$. We also calculated the density of states:

$$
N(\omega)=\sum_{\mathbf{k}} A(\mathbf{k}, \omega)
$$

Having obtained the spectral function we can calculate different physical properties of the cuprates. The real part of the $a b$-plane optical conductivity is given by:

$$
\sigma_{a b}(\nu)=\sigma_{0} \int d \omega \frac{f(\omega)-f(\omega+\nu)}{\nu} \sum_{\mathbf{k}}\left[\left(\frac{\partial \varepsilon_{\mathbf{k}}}{\partial k_{x}}\right)^{2}+\left(\frac{\partial \varepsilon_{\mathbf{k}}}{\partial k_{y}}\right)^{2}\right] A(\mathbf{k}, \omega) A(\mathbf{k}, \omega+\nu)
$$

where $\sigma_{0}=e^{2} \pi / 2 h$.

The in-plane conductivity $\sigma_{a b}(\omega)$ given by eq. (16) neglects the vertex correction. The in-plane conductivity is coherent in character and shows a Drude peak at low frequencies.

For the calculation of $c$-axis conductivity $\sigma_{c}(\omega)$ we consider the incoherent case. Incoherent conductivity corresponds to diffusive $c$-axis transmission and amounts in taking the averages of the spectral function $N(\omega)$ over all momenta.

$$
\sigma_{c}(\nu)=\sigma_{0} \int d \omega \frac{f(\omega)-f(\omega+\nu)}{\nu} \sum_{\mathbf{k}} t_{c}^{2}(\mathbf{k}) N(\omega) N(\omega+\nu)
$$

The same relation appear in the calculation of optical conductivity in disordered systems [31]. We calculate in the limit $\omega \rightarrow 0$ the in-plane resistivity $\rho_{a b}=\lim _{\omega \rightarrow 0}\left(1 / \sigma_{a b}(\omega)\right)$ and $c$-axis resistivity $\rho_{c}=\lim _{\omega \rightarrow 0}\left(1 / \sigma_{c}(\omega)\right)$.

In order to evaluate the thermopower we have to calculate [32]:

$$
L_{i j}=\int d \omega\left(-\frac{\partial f(\omega)}{\partial \omega}\right)\left[\sum_{\mathbf{k}}\left(\frac{\partial \varepsilon_{\mathbf{k}}}{\partial k_{x}}\right)^{2} A^{2}(\mathbf{k}, \omega)\right]^{i} \omega^{j-1}
$$

and the thermopower can be calculated using:

$$
S=-\frac{k_{B}}{|e| T} \frac{L_{12}}{L_{11}}
$$




\section{Results}

\subsection{Spectral Function and Phase Diagram}

In this section we present the obtained results for spectral function, using the self-consistent T-Matrix approximation described in previous section. In order to make the results more transparent we present the lowest order calculation results and the self-consistent calculations. We also present the phase diagram obtained for this model. As we can see both in Fig. 1 and Fig. 2 in the lowest order calculation we obtain a pseudogap along the direction $(0, \pi)-(\pi, \pi)$ and no pseudogap is obtained along the $(0,0)-(\pi, \pi)$ direction. This is in agreement with ARPES measurements [33]. Janko et.al. claims that this anomaly is caused by nearly stable cooper pairs in the excited states [34]. The same behavior is observed in the self-consistent calculation as we can see in Fig. 3 and Fig. 4. Qualitatively we found small differences in behavior between lowest and self-consistent calculation for the spectral function.

We also calculated the critical temperature $T_{c}$ of the system using Thouless criterion [16]. When $1+$ $V \Pi(\mathbf{k}, \omega)=0$ the superconductivity occurs. Thouless criterion is equivalent to BCS theory in the weak coupling limit. In our approximation $T(\mathbf{k}, \omega)$ can be regarded as the propagator of the Cooper pairs. Thouless criterion correspond to the situation in which $T(\mathbf{k}, \omega)$ has its pole at $\mathbf{k}=0$ and $\omega=0$. We are interested in the behavior of the system in the normal state, near the superconducting critical point, where the superconducting fluctuations are inhaced.

In Fig. 5 we present the phase diagram of the model where $T_{c}$ and $T_{M F}$ are the critical temperature calculate in the self-consistent and lowest-order calculated using Thouless criterion. We also calculated the critical temperature in the first order of perturbation theory and found that in the limit of strong coupling the self-consistent calculation of $T_{C}$ reach the limit of first order calculation of $T_{C}$. $T_{M F}$ can be viewed as the BCS critical temperature. The phase diagram corresponds to the underdoped region with the hole concentration $\delta=0.1$. Roughly speaking the pseudogap phenomena occurs in the region between $\mathrm{T}_{c}$ and $\mathrm{T}_{M F}$. This region is extremely wide in the strong coupling case. As we can see from the shape of spectral function $A(\mathbf{k}, \omega)$ the main effect of the electron-fluctuation interaction is the change of shape of $A(\mathbf{k}, \omega)$ which is seen near $\mathbf{k}=(0, \pi)$. This suggests that the electron-fluctuation interaction shifts the Fermi surface to the place far from $\mathbf{k}=(0, \pi)$. Similar with other reported self-consistent treatments of the T-matrix [35, 36] the pseudogap is not seen clearly in the integrated density of states but only in the k-dependent spectral function at extreme parameters ranges. In Fig. 6a we present the density of states for different couplings at the temperature $T=0.35 t$ and in Fig. $6 \mathrm{~b}$ the temperature dependence of the density of states for fixed coupling strength $V=-3 t$. As we can see from Fig. 6a, increasing the coupling strength, the density of states near $\omega=0$ slowly decreases but the central peak still remain. The central peak is due to the integration over the direction $(0,0)-(\pi, \pi)$ in the spectral function where no pseudogap appears. For the same temperature the central peak remain at the same frequency $\omega \approx 0$. Decreasing the temperature the central peak start to shifts to lower frequencies and a hump start to appear in the density of states. The two humps appearing in Fig. 6a are due to the pseudogap formation in the spectral function near the $(0, \pi)$ and symmetry related points and their intensity increase with lowering the temperature.

\subsection{Optical Conductivity and Resistivity}

We have calculated the in-plane and $c$-axis optical conductivity using eqs. (16) and (17). We also have calculated the resistivity as function of temperature for both in-plane and $c$-axis direction. Our approximation assumes the independent electron propagation in each layer and is justified for small interlayer hopping. The interlayer hopping hopping matrix element $t_{c}$ is weak resulting in an incoherent c-axis transport. Many approaches have been suggested [37, 38, 39] to describe the c-axis transport properties. The optical conductivity was calculated combining well controlled analytical and numerical methods in the case of one-dimensional Mott-Hubbard insulator at zero temperature in [40]. The $c$-axis optical conductivity and 
d.c. resistivity was calculated within $t-J$ model in 41.

In Fig. 7 we present the obtained results for the in-plane conductivity as function of frequency for two temperatures. The in-plane spectrum of $\sigma_{a b}(\omega)$ is dominated by Drude peak at $\omega=0$. The intensity of the peak grows by increasing the temperature. In Fig. 8 we present the $c$-axis optical conductivity for three temperatures at the doping level $\delta=0.1$. The electronic contribution to $\sigma_{c}(\omega)$ in the normal state of the underdoped cuprates is nearly $\omega$ independent and does nor form a Drude peak at $\omega=0$, indicating incoherent nature of the charge dynamics along the $c$-axis [42]. It should be emphasize that the pseudogap appears only in $\sigma_{c}(\omega)$. Actually the development of the pseudogap in $\sigma_{c}(\omega)$ is correlated with a rapid narrowing of Drude width in $\sigma_{c}(\omega)$. We observe an anomalous broad peak in $\sigma_{c}(\omega)$ at finite frequencies which grows in intensity as the temperature is lowered with no discontinuity at the superconducting transition similar to the experimental results [43]. In Fig. 9 we present the temperature dependence of the in-plane resistivity $\rho_{a b}$. We found a linear behavior for the in-plane resistivity at high temperature and a deviation from linearity below a characteristic temperature $T^{*}$. $T^{*}$ may be defined by different methods 44. In this paper we consider the temperature $T^{*}$ the characteristic temperature where the resistivity present a downturn, with a linear behavior above it. The arrows in Fig. 9 indicate $T^{*}$. Increasing the doping concentration $\delta, T^{*}$ decrease.

In Fig. 10 we present the $c$-axis resistivity. We found in this case the same linear behavior of resistivity as function of temperatures, at high temperatures and a upturn at lower temperatures $\left(d \rho_{c} / d T<0\right)$ signaling a semiconductor -like behavior. The semiconducting $T$ dependence of the $c$-axis resistivity is associated with the deepening of the pseudogap. Therefore the contribution to the conductivity from the quasiparticles near the $(\pi, 0)$ is suppressed by the pseudogap. The in-plane conductivity is determined by the contributions from "cold-spots" and the pseudogap is not important for the in-plane conductivity, but has more effect on the $c$ axis conductivity. The $c$-axis conductivity is determined from the contributions from the "hot-spots" where the pseudogap is large. Therefore the $c$-axis conductivity is remarkably suppressed by the pseudogap. This is in agreement with the experimental results for cuprates [45]. Our results are qualitatively in agreement with the experimental results obtained for $\left(R_{1-x} C a_{x}\right) B a_{2} C u_{3} O_{6+\delta}$ where with $C a$ doping the number of holes in $\mathrm{CuO}_{2}$ planes increases [46, 47], where $R$ stands for $Y$ and $L u . \rho_{a b}$ is linear in $T$ above $T^{*}$ and decreases more steeply below $T^{*}[24]$.

\subsection{Thermopower}

Thermopower has become a simple and ideal probe for the studies of high $T_{C}$ superconductors. The thermopower is one of the several physical quantities which distinctly reveals the unusual normal state properties. The results obtained for the thermopower are calculated using eq. (19). The thermopower of cuprates 448 has the propriety that is not a monotonic function of temperature. Several authors have tried to explain the temperature behavior of thermopower in term of phonon drag, metallic diffusion, semiconducting model and others [49], but they cannot explain the linear behavior at high temperature [50] without considering the cylindrical Fermi surface [51].

In Fig. 11 we present the temperature dependence of the thermopower. It can be seen that the normal state thermopower decrease with increasing hole doping $\delta$ to a lower positive value, at a fixed temperature. The temperature dependence of thermopower is linear with a negative slope at high temperature and present a broad peak, shifted toward the low-temperature as the doping increase 55. The hump of thermopower in the normal state was reported as a kind of universal behavior of HTSC, corelated with the opening of the pseudogap in the normal state of a superconductor [53].

In Fig. 12 we present the doping dependence of the thermopower at the interaction strength $V=-5.5 t$ and at the temperature $T=2 t$. At a fixed temperature much larger than the critical temperature, $S$ continuously decreases from underdoped to overdoped regions, changing its sign near optimal doping. The theoretical work presented in [54] shows that the doping dependence of the thermopower can be explained 
by the common band dispersion relation of high- $T_{c}$ cuprates. Therefore the thermopower can be a criterium for measuring the hole doping in $\mathrm{CuO}$ layers [55].

\section{Conclusions}

In this paper we have shown that the d-wave pairing scenario, based on the strong coupling superconductivity well explains some of the physical properties of the layered cuprates above the critical temperature $T_{c}$. We have considered that the strong coupling superconductivity takes place in underdoped cuprates and the pseudogap phenomena are its precursor.

We considered the T-Matrix approximation which is the dominant scattering vertex in the vicinity of the superconducting critical point. In this analysis we didn't considered the vertex corrections. Anyway our theory appropriately explains the pseudogap phenomena in high- $\mathrm{T}_{c}$ cuprates. We did not take care into account the antiferromagnetic spin fluctuations which are important for explaining other properties of cuprates such as the NMR spin lattice relaxation $1 / T_{1}$.

Based on this scenario we studied charge dynamics, in-plane and $c$-axis resistivity and thermopower and compared the results with the experimental results. As can be seen from previous section our theory fit well the experimental results from $Y B C O$ class of layered superconductors. For the calculation of in-plane charge dynamics we obtained a spectrum dominated by a Drude term, while for $c$-axis dynamics we found an almost frequency independent behavior. For the $c$-axis resistivity we found, due to the opening of the pseudogap in the density of states, a semiconductor-like behavior. For the temperature dependence of thermopower we found a linearly dependence at high temperatures with a negative slope. The negative slope depends weakly on the doping level.

\section{Acknowledgments}

The research was supported by the CNCSIS contract no. 3447/1999.

\section{References}

[1] M. Imada, A. Fujimori and Y. Tokura Rev.Mod.Phys. 701039 (1998)

[2] T. Dahm, D. Manske and L. Tewordt Phys.Rev. B55 15274 (1997)

[3] M. Randeria cond-mat/9710223

[4] V.J. Emery, S.A. Kivelson and O. Zachar Phys. Rev. B56 6120 (1997)

[5] H. Fukuyama, H. Kohno, B. Normand and T. Tanamoto J. Low. Temp. Phys. 99429 (1995)

[6] S. Doniach and M. Inui Phys. Rev. B41 6668 (1990)

[7] V.J. Emery and J.R. Kivelson Nature 374434 (1995)

[8] Z.X. Shen and J.R. Schriffer Phys.Rev.Lett 781771 (1997)

[9] J. Schmalian, D. Pines and B Stojkovic Phys. Rev. Lett. 803839 (1998)

[10] A.V. Chubukov cond-mat/9709221

[11] R.S. Markiewicz et. al. Phys. Rev. B60 627 (1999) 
[12] Z. Dai et. al. Phys. Rev. B48 14543 (1993)

[13] Y. Yanase and K. Yamada cond-mat/0002274

[14] M. Crisan, C. P. Moca and I. Tifrea Phys.Rev. B59 14680 (1999)

[15] G. Preosti, Y.M. Vilk and M.R. Norman Phys.Rev. B59 1474 (1999)

[16] P. Nozieres and S. Schmitt-rink J. Low. Temp. Phys. 59195 (1985)

[17] J. Maly, B. Janko and K. Levin Physica C 32113 (1999), cond-mat/9805018

[18] B.G. Levi Phys. Today 4617 (1993)

[19] Z.X. Shen Phys. Rev. Lett. 701553 (1993)

[20] D.S. Marshall et.al. Phys.Rev.Lett. 764841 (1991)

[21] M. Oda et.al. Physica C 281135 (1997)

[22] V.M. Krasnov et. al. Phys. Rev. Lett. 845860 (2000)

[23] S.Uchida Physica C 282-287 12 (1997)

[24] T. Ito, K. Takenaka and S. Uchida Phys. Rev. Lett. 703995 (1995)

[25] K.Takenaka et.al Phys. Rev. B50 6534 (1994)

[26] S. Chakravarty et.al Science 261337 (1993)

[27] O.K. Andersen et.al. J. Phys. chem. Solids 561573 (1995)

[28] A. Yurgens et.al. cond-mat/9907159, to be published in Int. J. Mod. Phys. B

[29] B. Kyung et.al. Phys. Rev. Lett. 803109 (1998)

[30] H. Vilberg and J. Serene J. Low. Temp. Phys. 29179 (1997)

[31] N.F. Mott and E. a. Davis electronic proceses in non-crystalline materials, Clarendon Press 1979

[32] J. Merino and R.H. McKenzie cond-mat/9909041

[33] H. Ding et.al. Nature 38251 (1996), M.R.Norman et.al. Nature 392157 (1998)

[34] B. Janko, J. Maly and K. Levin Phys. Rev. B56 11407 (1997)

[35] M. Keller, W. Metzner and U. Schollwock Phys. Rev. B60 3499 (1999)

[36] B. Kyung, E.G. Klepfish and P.E. Kornilovitch Phys. Rev. Lett. 803109 (1998)

[37] P.W. Anderson The Theory of Superconductivity in the High- T $_{c}$ Cuprates (Princeton university Press,Prinston 1997)

[38] A.J. Leggett Phys. Rev. Lett. 83392 (1999)

[39] S.L.Cooper and K.E. Gray in Physical Properties of High-Temperature Superconductors IV (World Scientific, Singapore 1994) 
[40] E. Jeckelmann, F. Gebhard and F.H.L. Essler cond-mat/9911281

[41] P.Prelovsek, A. Ramsak and I. Sega Phys. Rev. Lett. 813745 (1998)

[42] K. Tamasaku et.al. Phys. Rev. Lett 723088 (1994)

[43] T. Timusk and B. Statt cond-mat/9905219

[44] J.L. Tallon and J.W. Loram to be publised

[45] Y. Nakamura and S. Uchida Phys.Rev.B47 8369 (1993)

[46] T. Watanabe, M. Fujiwara and N. Suzuki Physica C 252100 (1995)

[47] R. Pinto et.al. Physica C 289280 (1997)

[48] J.S. Zhou and J.B. Goodenough Phys.Rev. B51 3104 (1995), Phys. Rev. B54 13393 (1996)

[49] A.B. Kaiser and C. Uher in Studies of High Temperature Superconductors, vol. 7, (Nova Science Publishers, New York 1990)

[50] T. Obtani and K. Ohkuma Solid State Commun.72 767 (1989)

[51] H.J. Trodah Phys.Rev B51 6175 (1995)

[52] K.Matsuura et.al. Phys. Rev. B46 11923 (1992)

[53] J.L.Tallon et.al. Phys. Rev. Lett. 75, 4114 (1995)

[54] Y. Kubo Phys. Rev. B50 3181 (1994)

[55] S.D. Obertelli, J.R. Cooper and J.L. Tallon Phys. Rev. 4614928 (1992) 


\section{Figure Captions}

Figure 1

Spectral function in the lowest order approximation at $T=0.6 t$ in the $(0, \pi)-(\pi, \pi)$ direction at the doping concentration $\delta=0.1$ and with the coupling strength $V=-5 t$

Figure 2

Spectral function in the lowest order approximation at $T=0.6 t$ in the $(0,0)-(\pi, \pi)$ direction at the doping concentration $\delta=0.1$ and with the coupling strength $V=-5 t$

Figure 3

Spectral function in the self consistent approximation at $T=0.6 t$ in the $(0, \pi)-(\pi, \pi)$ direction at the doping concentration $\delta=0.1$ and with the coupling strength $V=-5 t$

Figure 4

Spectral function in the self consistent approximation at $T=0.6 t$ in the $(0,0)-(\pi, \pi)$ direction at the doping concentration $\delta=0.1$ and with the coupling strength $V=-5 t$

Figure 5

Phase diagram of the model at hole concentration $\delta=0.1$

Figure 6 a

Density of states for temperature $T=0.35 t$ and for different coupling strength. The solid line correspond to $V=-t$, the dotted line to $V=-3 t$ and the dashed line to $V=-6 t$. The hole concentration is $\delta=0.1$.

Figure $6 \mathrm{~b}$

Density of states for different temperatures and for coupling strength $V=-3 t$. The solid line correspond to $T=3.5 t$ and the dashed line to $T=0.35 t$ at hole concentration is $\delta=0.1$.

Figure 7

In-plane optical conductivity at $T=0.4 t$ (solid line) and $T=3.4 t$ (dashed line) at the doping concentration $\delta=0.1$ and coupling strength $V=-5.5 t$

Figure 8

$c$-axis optical conductivity at different temperatures. $T=0.6 t$ (solid line) $T=1.0 t$ (dash line) $T=1.6 t$ (dot line) in the limit of small frequencies at the coupling strength $V=-5.5 t$ and doping level $\delta=0.1$

Figure 9

Temperature dependence of the in-plane resistivity at different densities, $\delta=0.1$ (solid line) $\left(T_{C}=0.21 t\right)$, $\delta=0.15$ ( dashed line) $\left(T_{C}=0.3 t\right)$ and $\delta=0.2$ (dashed-dotted line) $\left(T_{C}=0.36 t\right)$, at the same coupling strength $V=-5.5 t$. The arrows indicate the $T^{*}$.

Figure 10

Temperature dependence of the $c$-axis resistivity at different densities, $\delta=0.1$ (solid line) $\left(T_{C}=0.21 t\right)$, $\delta=0.15$ ( dashed line) $\left(T_{C}=0.3 t\right)$ and $\delta=0.2$ (dotted line) $\left(T_{C}=0.36 t\right)$, at the same coupling strength $V=-5.5 t$.

Figure 11

Temperature dependence of thermopower at different densities, $\delta=0.1$ (solid line) $\left(T_{C}=0.21 t\right), \delta=0.15$ ( dashed line) $\left(T_{C}=0.3 t\right)$ and $\delta=0.2$ (dotted line) $\left(T_{C}=0.36 t\right)$, at the same coupling strength $V=-5.5 t$.

Figure 12

Termopower as function of the doping concentration $\delta$ at the coupling strength $V=-5.5 t$ and $T=2 t$ 


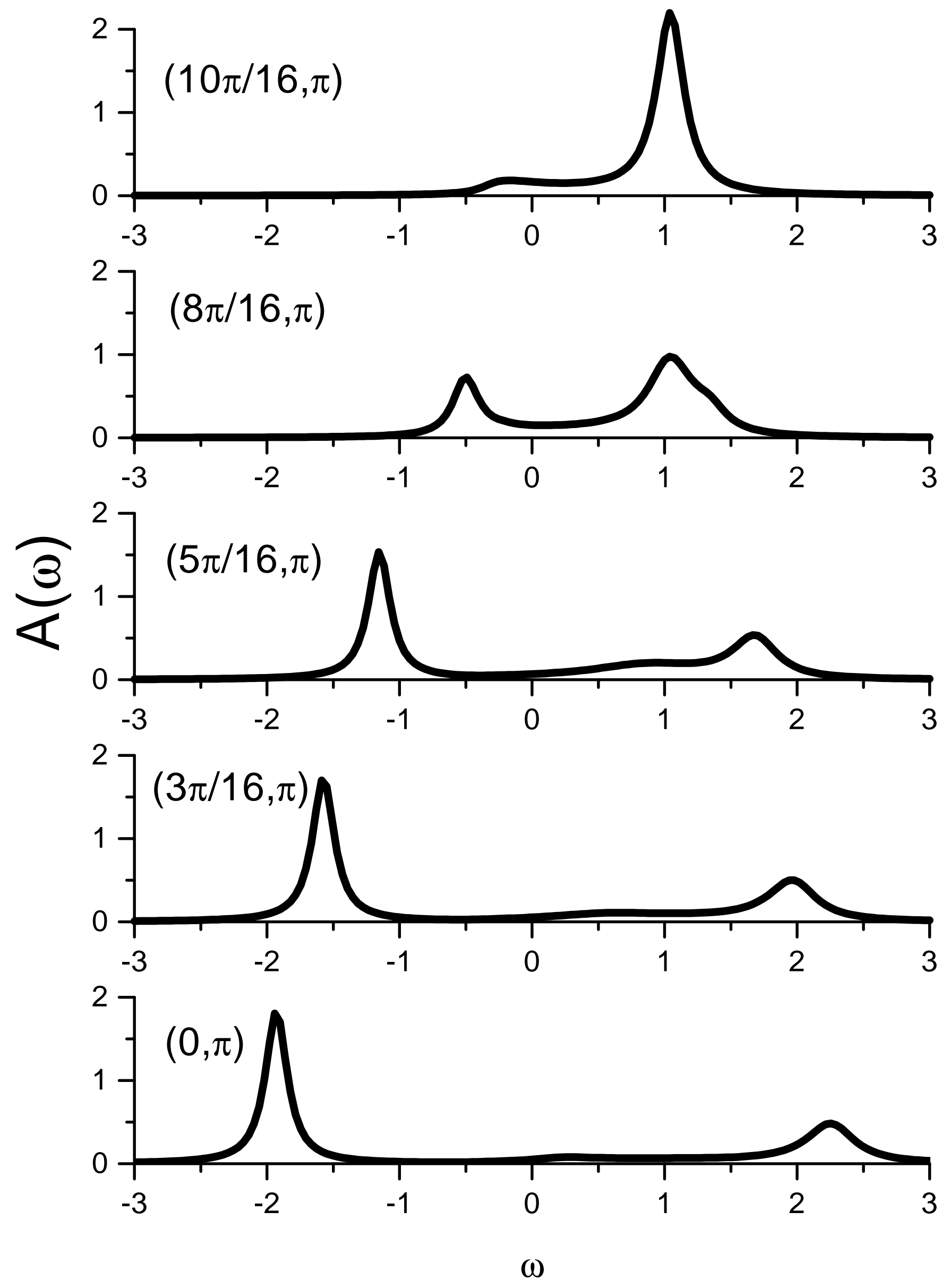

Fig. 1 

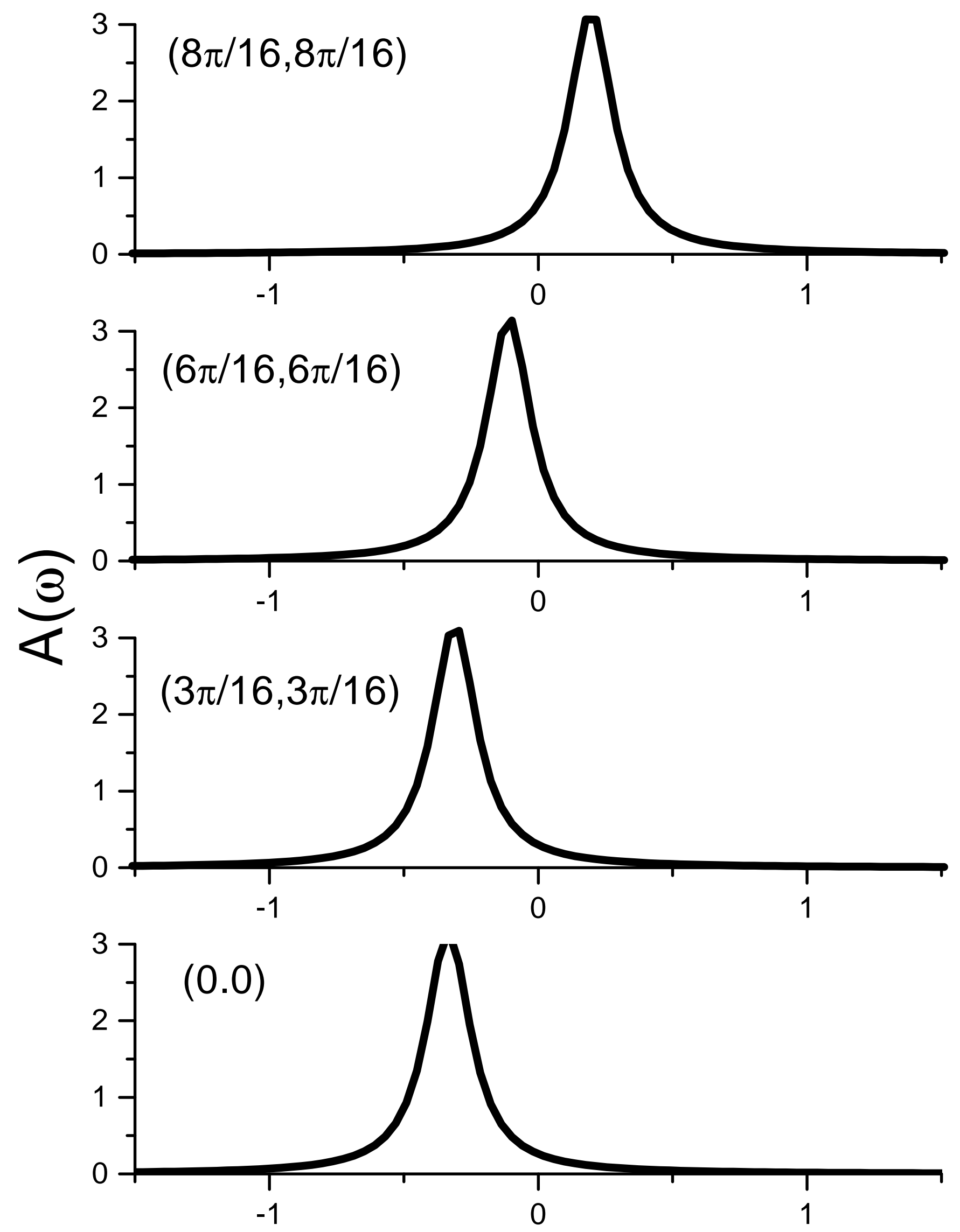

a)

Fig. 2 

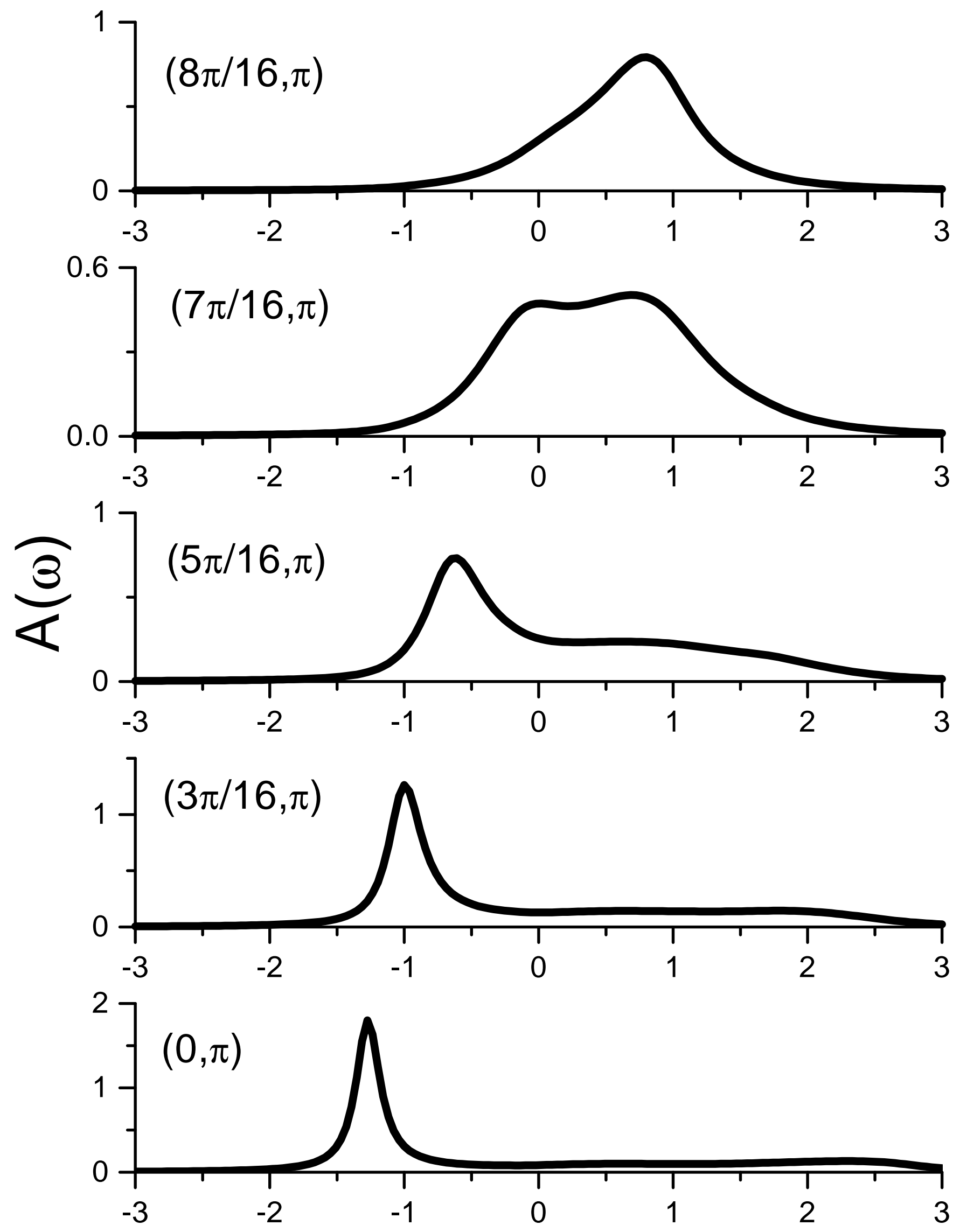

$\omega$

Fig. 3 

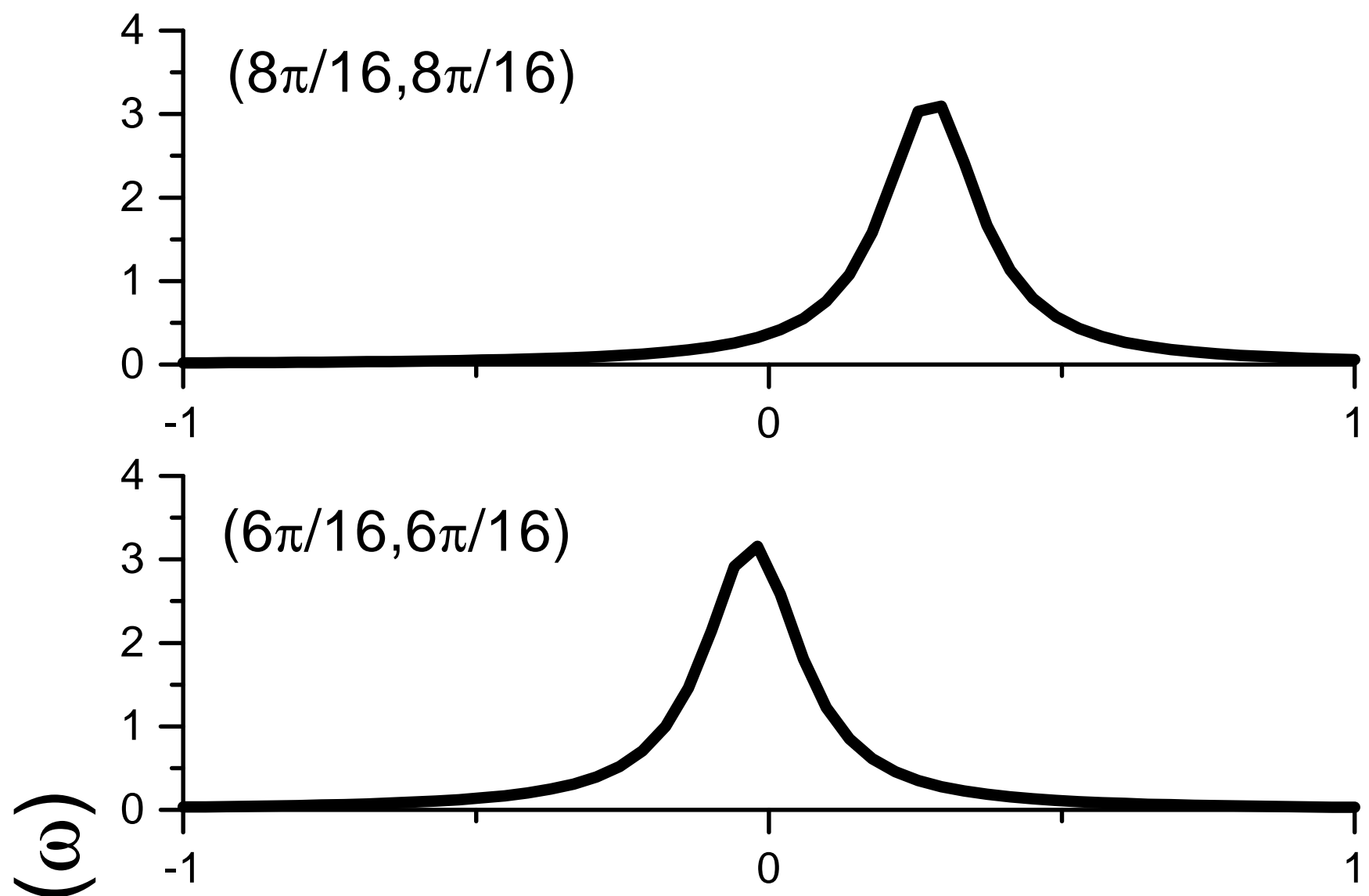

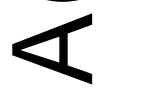
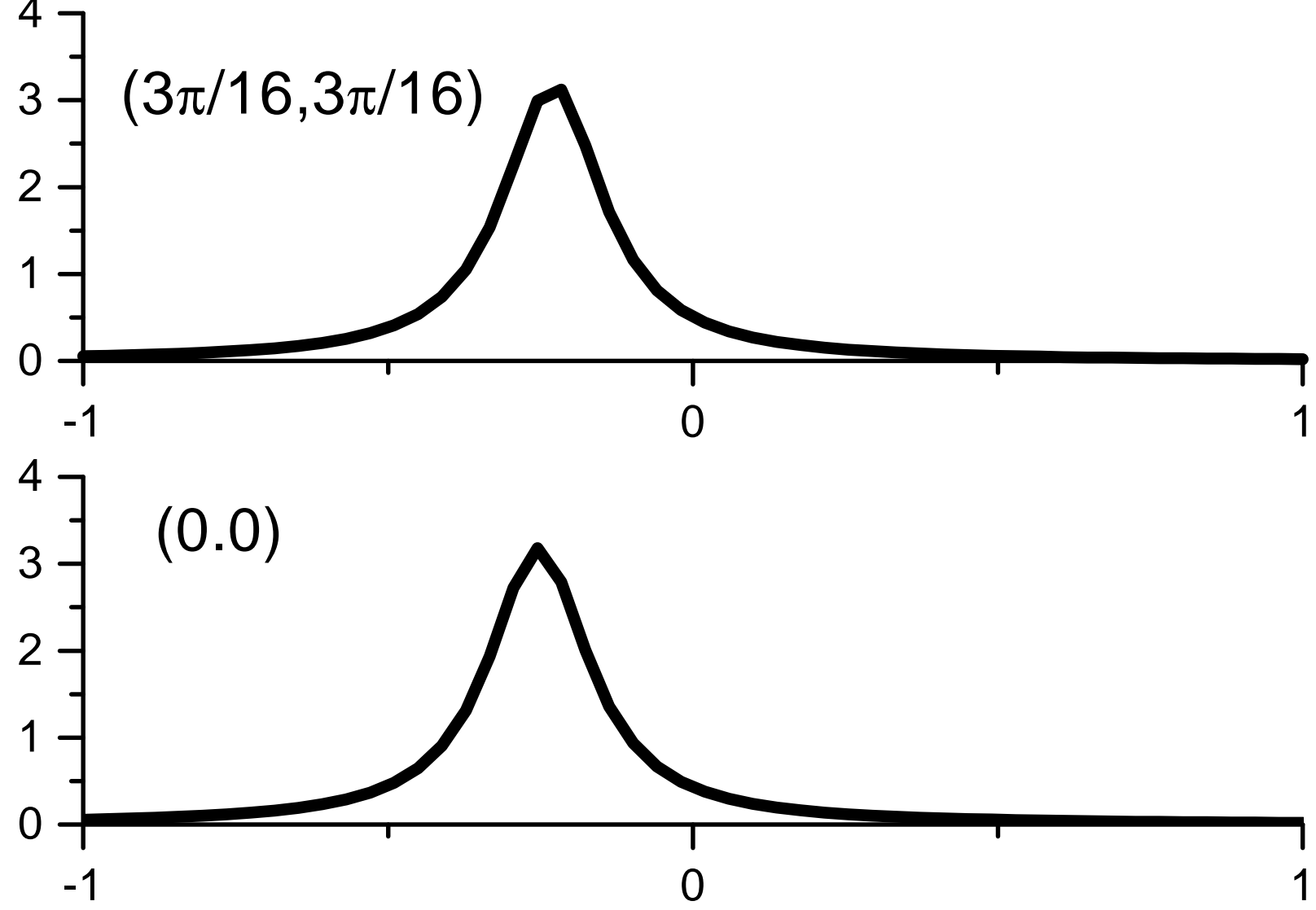

$\omega$

Fig. 4 


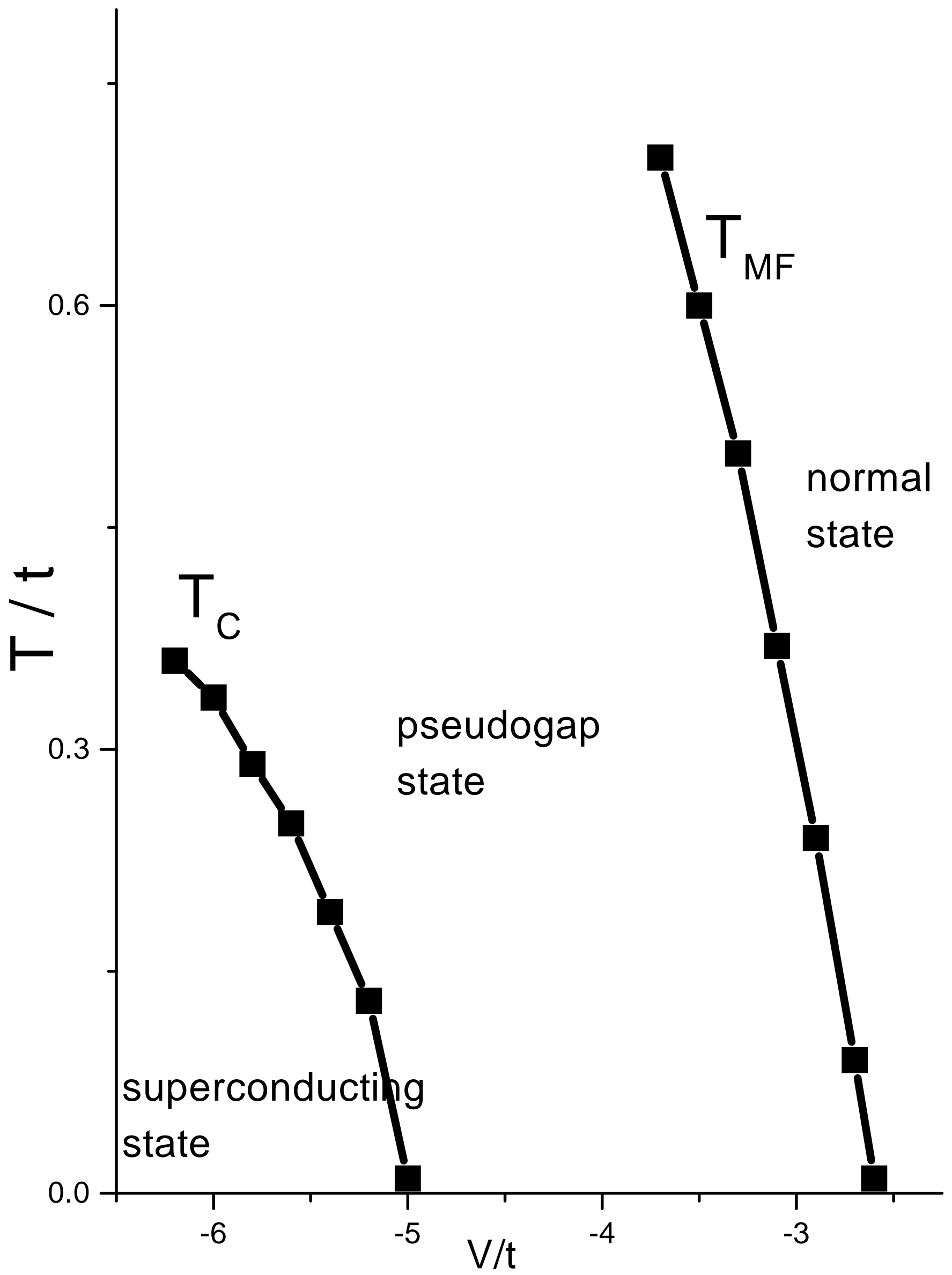

Fig. 5 


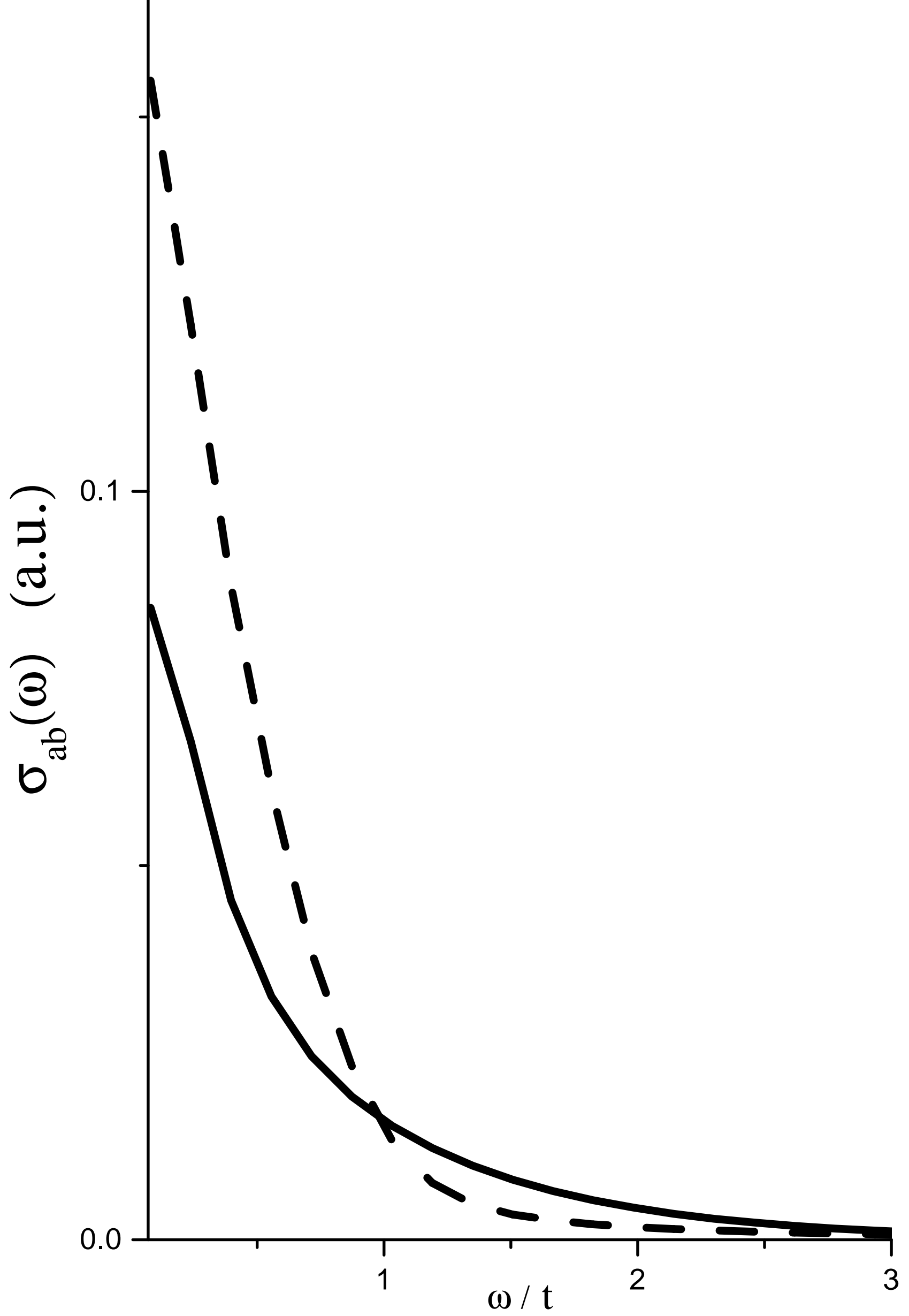

Fig. 7 


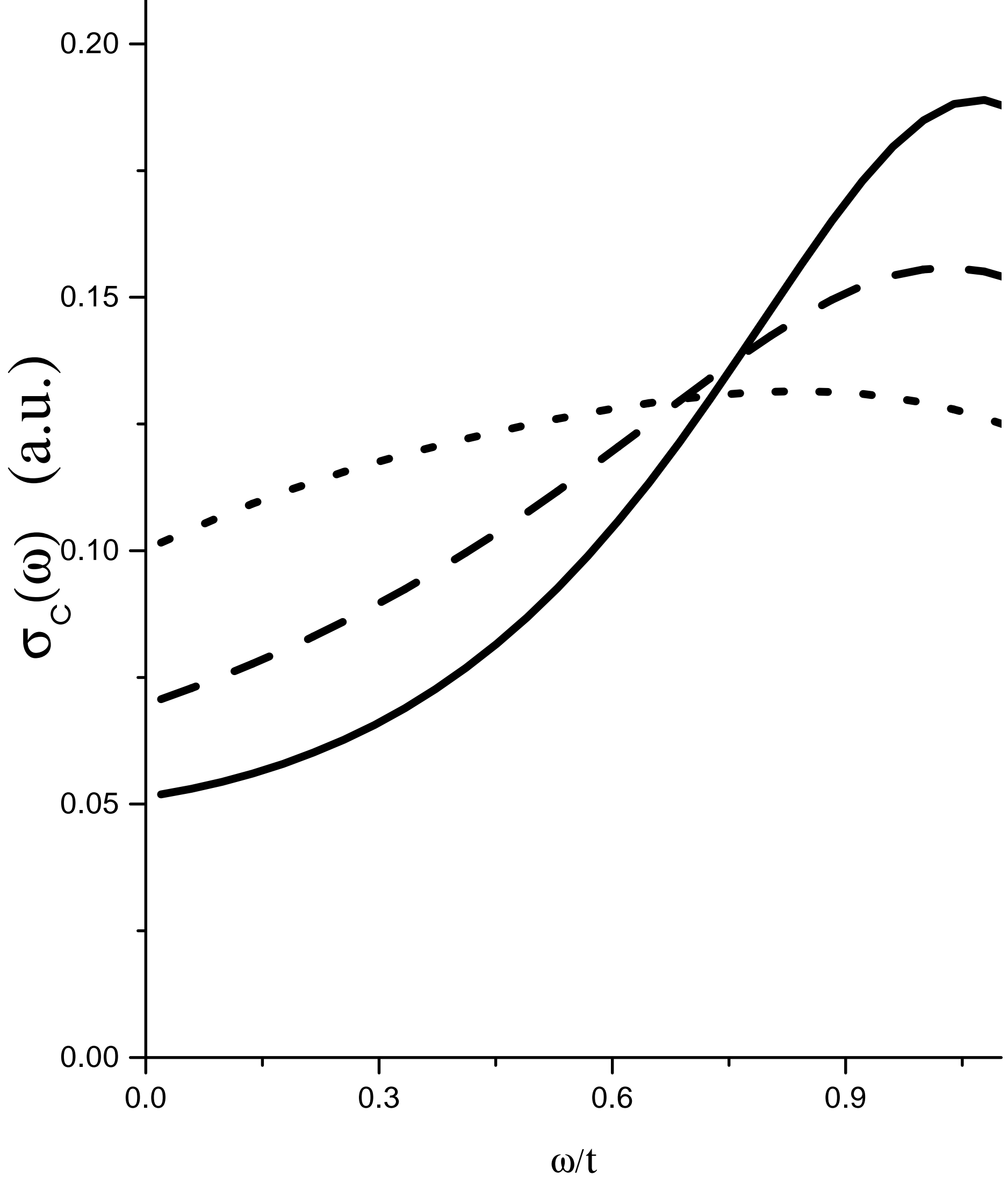

Fig. 8 


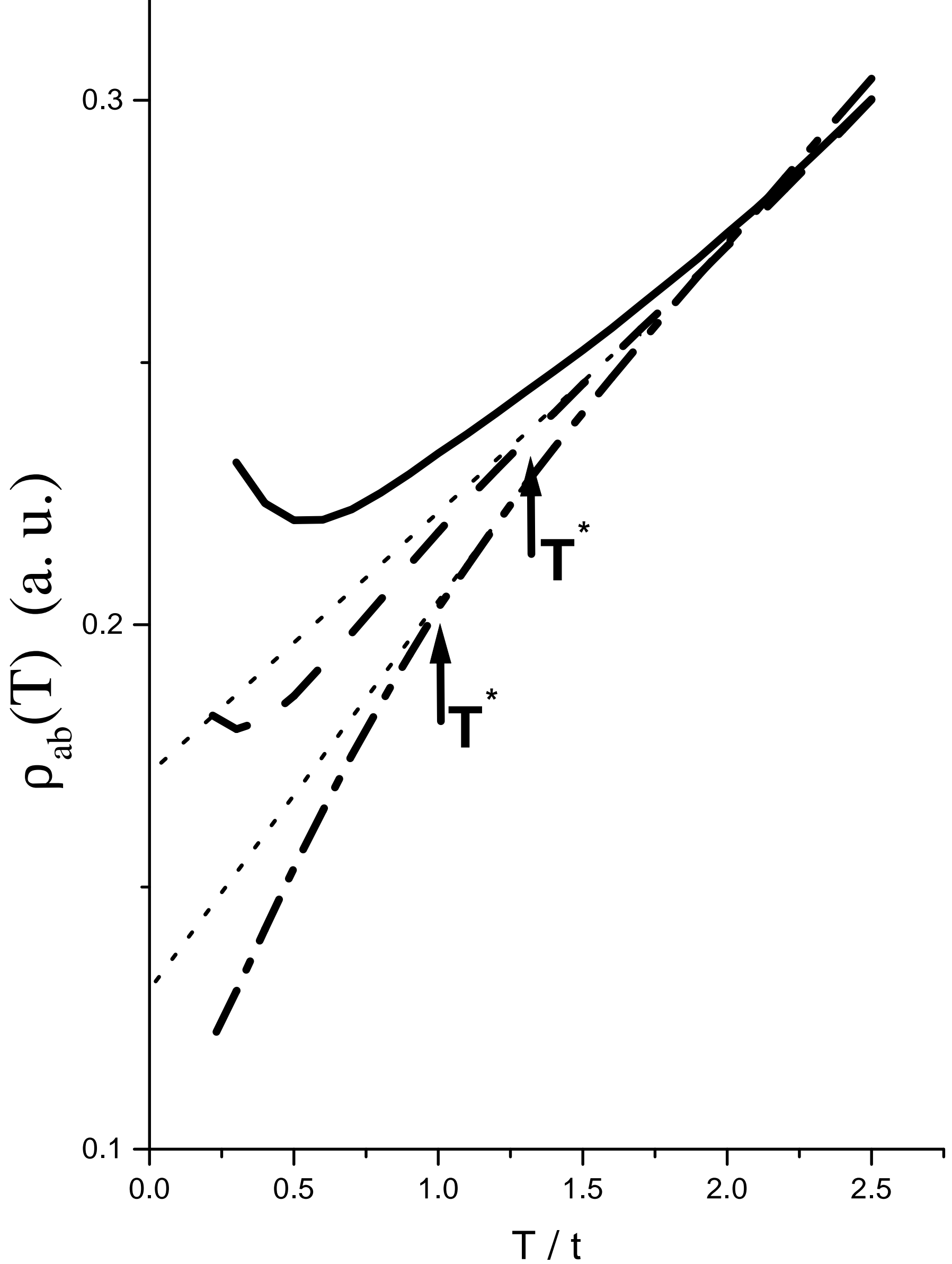

Fig. 9 


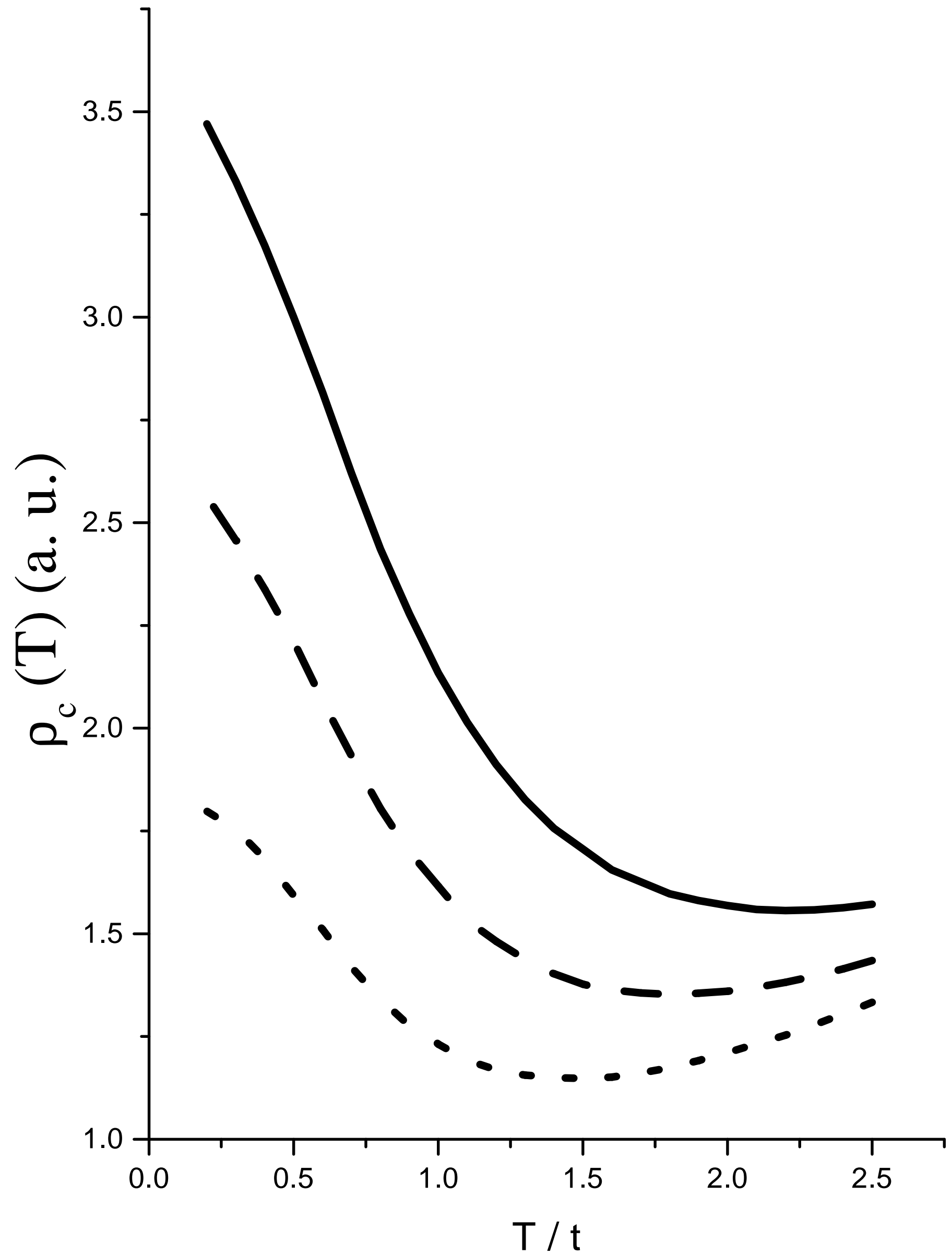

Fig. 10 


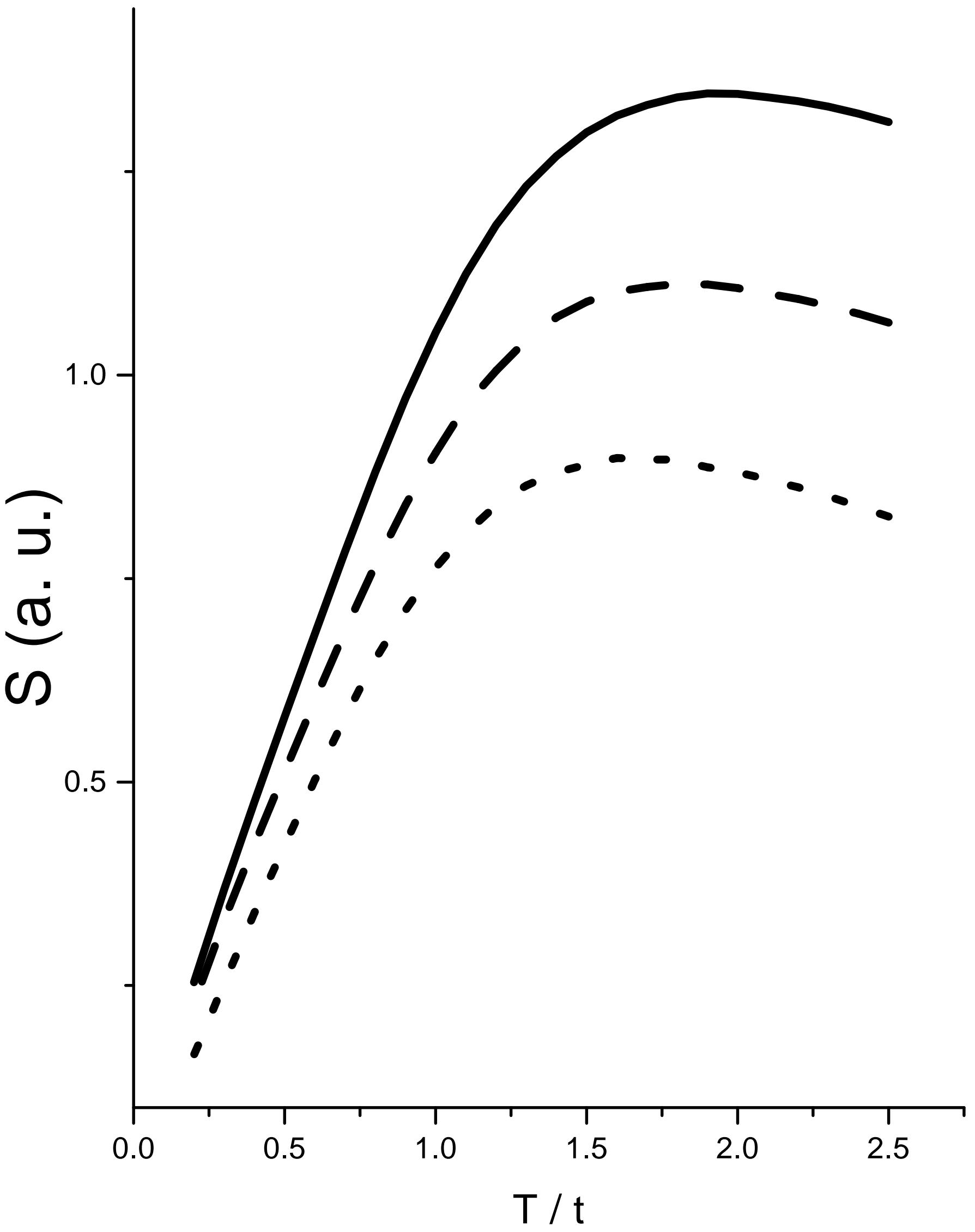

Fig. 11 


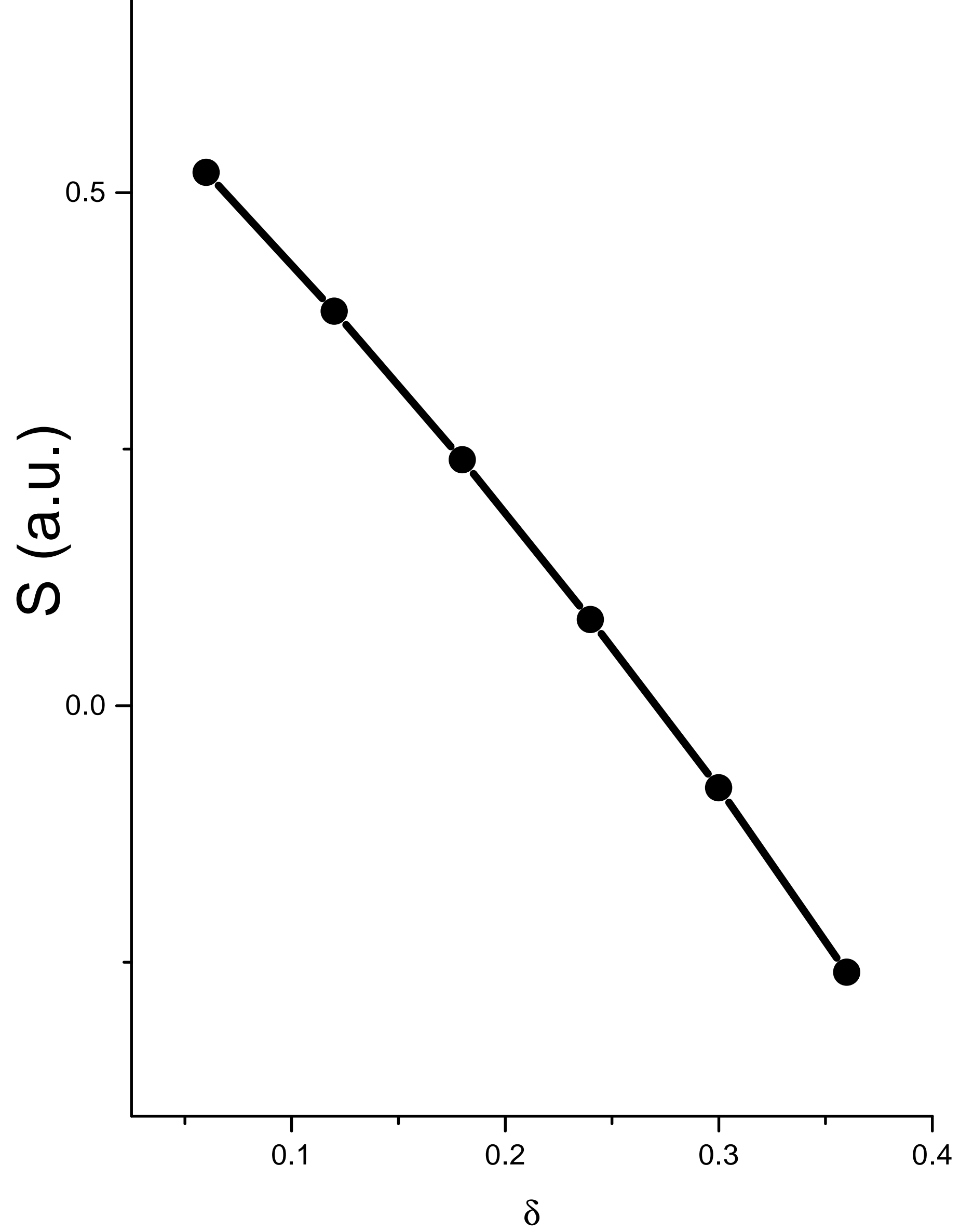

Fig. 12 